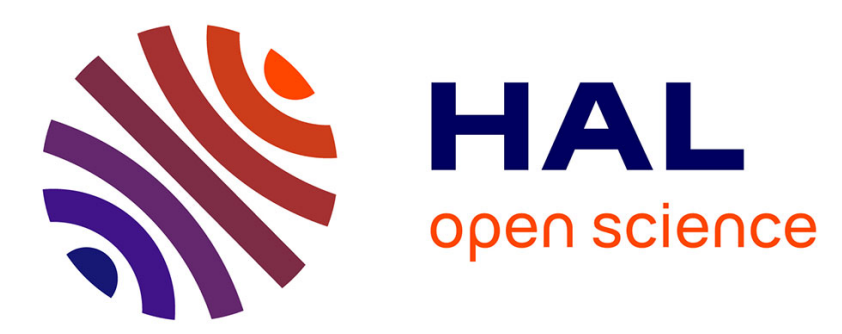

\title{
A Traceability Service to Facilitate RFID Adoption in the Retail Supply Chain
}

Gabriel Hermosillo, Julien Ellart, Lionel Seinturier, Laurence Duchien

\section{To cite this version:}

Gabriel Hermosillo, Julien Ellart, Lionel Seinturier, Laurence Duchien. A Traceability Service to Facilitate RFID Adoption in the Retail Supply Chain. IWRT 2009 - 3rd International Workshop on RFID Technology - Concepts, Applications, Challenges IWRT 2009, INSTICC, May 2009, Milan, Italy. pp.49-58. inria-00391089

\section{HAL Id: inria-00391089 \\ https://hal.inria.fr/inria-00391089}

Submitted on 3 Jun 2009

HAL is a multi-disciplinary open access archive for the deposit and dissemination of scientific research documents, whether they are published or not. The documents may come from teaching and research institutions in France or abroad, or from public or private research centers.
L'archive ouverte pluridisciplinaire HAL, est destinée au dépôt et à la diffusion de documents scientifiques de niveau recherche, publiés ou non, émanant des établissements d'enseignement et de recherche français ou étrangers, des laboratoires publics ou privés. 


\title{
A Traceability Service to Facilitate RFID Adoption in the Retail Supply Chain
}

\author{
Gabriel Hermosillo, Julien Ellart, Lionel Seinturier, Laurence Duchien \\ INRIA Lille - Nord Europe - University Lille 1 - Laboratoire LIFL - CNRS UMR 8022 \\ \{firstName.lastName\}@inria.fr
}

\begin{abstract}
Nowadays, companies are suffering changes in the way they deal with their inventories and their whole supply chain management. New technologies are emerging to help them adapt to the changes and keep a competitive status, but the adoption of such technologies is not always easy. Even though a lot of research has been done for RFID, there are still some areas that are being left aside, like the traceability aspect, which is one of the most important concerns in the retail supply chain. We propose a service named TRASER (TRAceability SErvice for the Retail supply chain) that will help the companies adopt the new technologies into their existing environments, dealing with persistence and traceability, and allowing the users to manage their operation according to their business rules, workflows and historical data.
\end{abstract}

\section{Introduction}

Radio Frequency Identification (RFID) is a technology used to identify a tagged object using radio frequency. It is mainly composed of an electronic tag attached to an object, a reader that gathers the tag's information and a computer system connected that receives and processes the information provided by the readers [1]. RFID has come as a relay to the bar code systems, bringing several advantages such as not to require a line-of-sight between the tag and the reader, multiple tags read at once, possibility to hide the tag or make it part of the design. The tags can be read from a short distance up to considerably long distances (tens of meters with an active tag). Because of these advantages, RFID applications are growing widely in different domains, including health care and medicine $[2,3]$, warehouse management $[4,5]$, aerospace and automobile industry [6,7], construction and maintenance of oil facilities [8], retail and supply chain management $[9,10,11]$ and anti-counterfeit [12].

RFID has evolved during the last few years, from being an obscure technology, almost unknown, used only by a small group of companies, to a fundamental technology that is now adopted and used worldwide by many important companies, consumer applications and governments. In commerce, RFID technology is mainly used for monitoring inventory and identifying different assets (i.e. pallets, containers, etc.), while in the industry, it is mainly used for monitoring production processes. In either environment, RFID can provide immediate and tangible 
benefits throughout the supply chain. Rather than displacing bar codes, RFID will complement them, helping to facilitate all the supply chain logistics in the companies that adopt it.

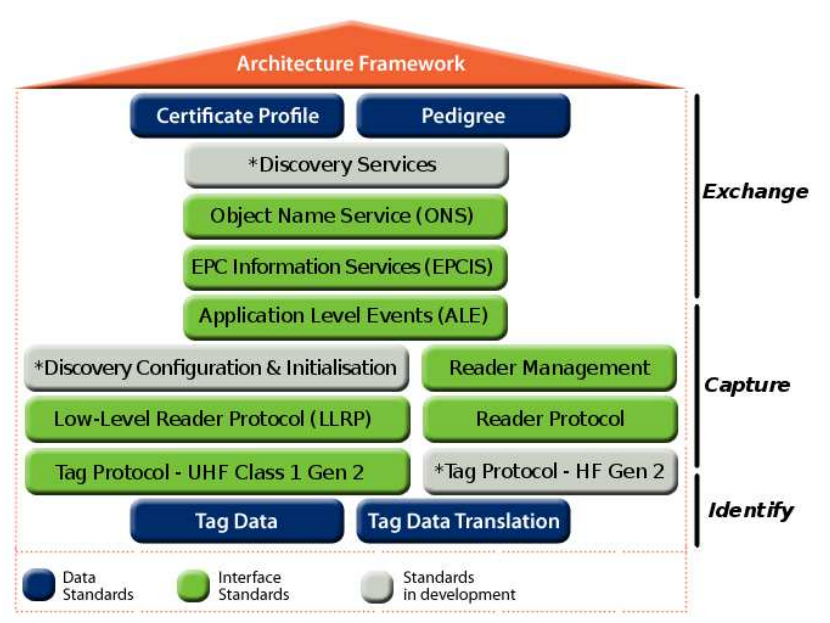

Fig. 1. EPCglobal's standards [1]

According to some authors, there are three main research streams in RFID applied to the supply chain: conceptual analysis of opportunities (benefits and risks), case studies, and business values [13]. Others say that research publications are mainly focused on technology, applications, and security [14]. The truth is that RFID is beginning to become a key part of the industry, and its adoption is eminent for those who want to keep a competitive status.

To correctly use and exploit the RFID technology, we need specific services that will allow us to take advantage of the gathered information and to use it in a business model. Some of these services are already defined by the EPCglobal's standards, like the use of ONS, EPCIS and ALE, as shown in Figure 1, but others are not so deeply considered, such as traceability and event storing. The lack of research and implementation of these services has made it harder for the industry to adopt RFID and integrate them to their existing applications, specially for the retail supply chain, where these approaches are some of the most important.

Our project intends to cover those needs. In the following sections we propose an RFID service named TRASER that will deal with persistence and traceability, allowing the users to manage their operations according to business rules, workflows and historical data. This service will be part of a whole platform, which has for objective to create a shared technical infrastructure, generic and parametrized, capable of managing the communication of heterogeneous systems (RFID, bar codes, NFC, etc.) among them and with existing IT services, to help the companies exploit and benefit from the current technologies. This research 
takes place within the ICOM project, funded by "Pôle de compétitivité" PICOM and the Région Nord-Pas-de-Calais in France.

This paper is organized as follows: Section 2 explains the motivation of the project. Section 3 describes our proposal. Section 4 presents some of the related work. Finally, section 5 concludes and discusses some future work.

\section{Motivation for a traceability service}

In the retail supply chain, it is important to know the amount of inventory held, where the products are located, in what condition they are and sometimes even to what environment they have been exposed. This information is usually gathered manually, and occasionally registered and then fed into a business application to be considered. The use of bar codes has been used to keep track of products and to relate them with the rest of the information, but unfortunately it is not very accurate. Bar codes are generic, so a reading of a bar code does not provide information about an individual product, but rather of a particular kind of products. That means that if we have 10 identical products, we can scan each one or just scan the same one 10 times and get the same result. This often leads to human errors during the stock receiving and inventory processes, where the employees just scan one product and multiply it by the number of identical products that they think they have, instead of scanning each product individually. Another problem is that if during the process one of the products is lost, we would not know for sure in which step of the process we lost it, because there is no individual tracking and normally the employee will not scan all the products again on each step of the process.

A solution for these problems is to use an RFID infrastructure, which will allow each tag to be considered individually, and then add a traceability service to get the information about the product's location and environment and complement the package with persistence, so that not only the present status of the product is considered, but also the historical data that has been gathered about it can be used. The process has to be relatively easy for the industry to adopt the RFID services, and it needs to have useful results and be quite simple to manage. In this context, the data contained in a tag is not interesting when it is isolated, but when it is used within a business application it becomes very important. Every information sent by the RFID or bar code readers creates an event, which can be used to go from one state of the process to another, but not all the events cause a state change, sometimes it is a group of events, or even the order in which they happen, what is needed to create such a transition.

An example of such transitions can be seen in Figure 2. If we have a product $X$ in a warehouse, when the $\operatorname{tag}$ of $X$ is read by reader $A$ in the exit of the warehouse, and a few minutes later it is read by reader $B$ in the sales floor, it will create a transition of product $X$ to be on sale, but if instead it is read by reader $C$ on the shipping area, the transition of product $X$ will go to shipping. So depending on the business process, the status of the product will change according to its location. 


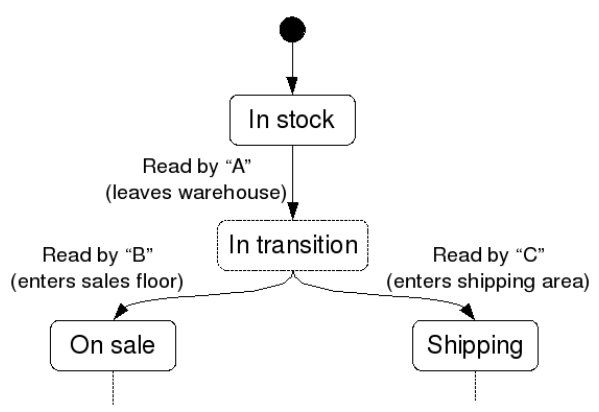

Fig. 2. Transition example

Gathering all the possible information from the created event, like cause, time and place, will help the final user to create a workflow that will fit better to their business process by adding Complex Event Processing (CEP) to the system [15]. This can even be used to create on-the-fly reports that are updated with each event and create alerts when an anomaly is found, which help the user to keep a live monitoring of the process. It is in this context that we propose $T R A S E R$, a traceability service for the retail supply chain, that will allow the companies to know the status of their products and incorporate that information into their business management.

\section{TRASER: a TRAceability SErvice for the Retail supply chain}

\subsection{An overview of TRASER}

The objective of TRASER is not to create a completely new approach to solve the problems, but to bring together the existing standards and research projects to create a complete and useful service that can be easily adopted in the retail supply chain. In order to understand how our system works, Figure 3 shows a basic architecture, based on the EPCglobal's standards [1]. The system receives the events created from the readers (after being filtered), containing the reader ID, the time of read and the Electronic Product Code (EPC) (step 1), among other fields that can be added according to the user's needs. These events will be translated to a business event (step 2), to have a more significant meaning. To increase the usefulness of the events, there is a need to store them so they can be available at any time and be considered for the decision making process or be consulted by any system interested in them, and to cover this need, we use the EPC Information System (EPCIS) repository (step 3), following the EPCglobal's standards [16]. For the external entities, it is not so easy to know where the information about a tag is being stored, so there must be a service which can provide the relation between a tag and the EPCIS repository containing it's information. Here is where the EPCglobal's architecture requires the Object 
Name Service (ONS) (step 3'), a service based on the Domain Name System (DNS) [16]. Finally, an application interface will allow the existing applications to exploit the generated information (step 4) or even send new business events through it (step 4'). This last step allows the generation of events that are not related to the readers (i.e. a manual intervention in a certain step of the process).

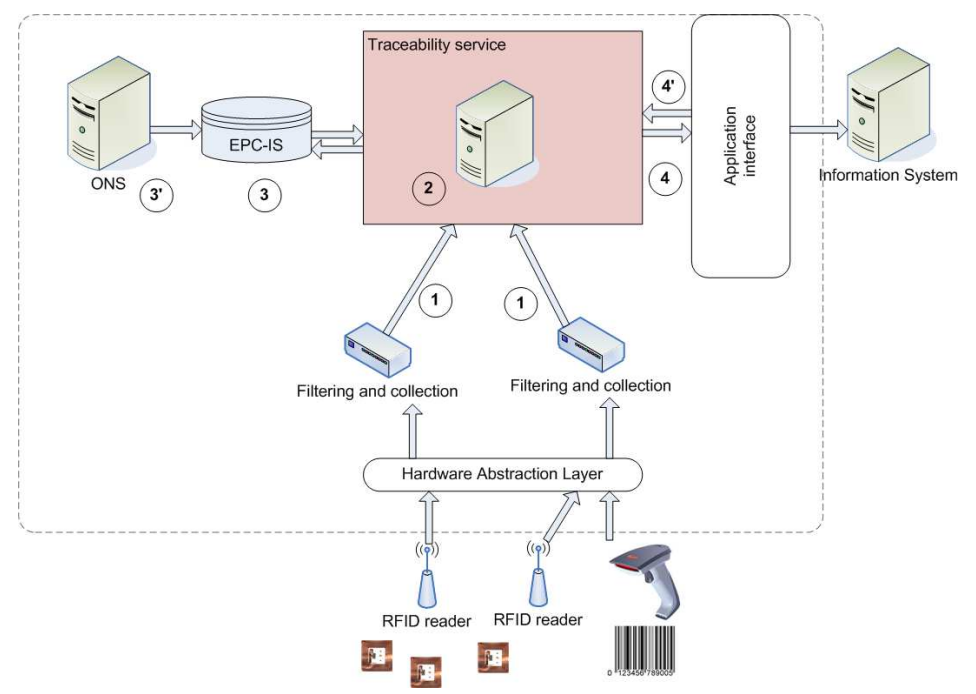

Fig. 3. System architecture

\subsection{Traceability service}

To simplify and improve the traceability service, we divided the whole process in two main parts: Event Translation, which will transform the ALE events into business events, using Complex Event processing (CEP) and a rule engine, and the Process Manager, which will receive the business event and create the transitions accordingly in the Business Process Manager (BPM), as shown in Figure 4. Both parts are explained in more detail in the following sections.

In the Event Translation part, the service has an Application Level Event (ALE) interface that receives the events from the readers after being filtered, or any other source that can generate an ALE event (like intelligent readers). The input of this interface shall be an XML file containing the fields and information specified in the EPCglobal's ALE standard 1.1 [16]. After that, the events are transformed into EPCIS events (or business events), using the business rules defined by the user. The EPCIS events are defined in the EPCglobal's EPCIS standard 1.0.1 [16], and are introduced into the system using the Core Business Vocabulary created by EPCglobal for a better, and yet standard, use and understanding of the events. Once the translation is done, the EPCIS events are 


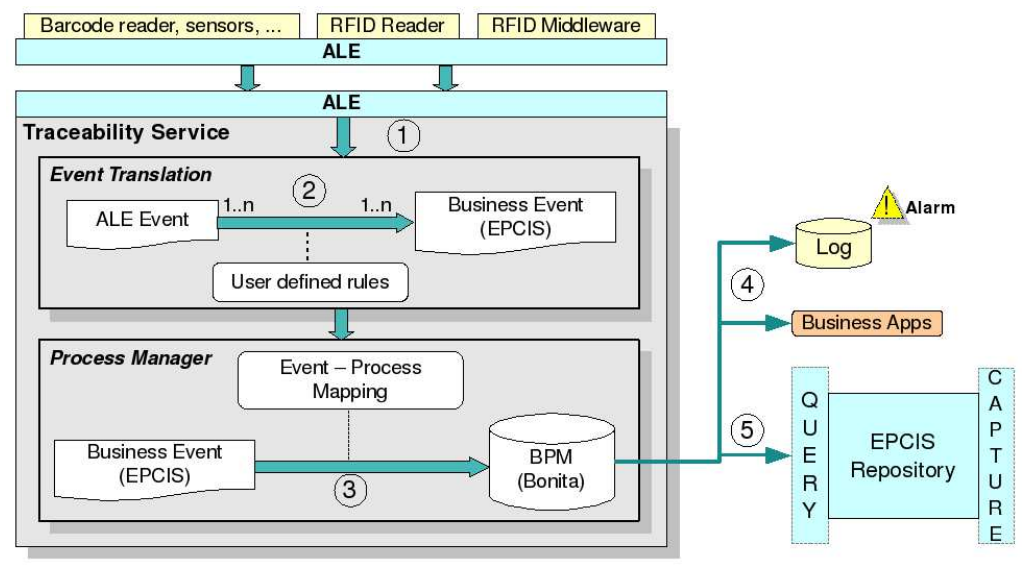

Fig. 4. Traceability service

then sent to the process manager, where they will be analyzed and will allow the process to move from one state to another, according to the provided workflow of the business. This will be done using a state-transition diagram, that will show the different states in which a tag (or the object containing the tag) will be followed. At the end, the process engine will trigger a new event for the business applications or an alert, in case some conditions are not met.

\subsection{Complex Event Processing}

In Complex Event Processing (CEP), the main goal is to figure out a meaningful and complex event by analyzing multiple streams of simple events. These complex events can be either the occurrence of a group of simple events in any order or the sequence of them [15]. An example of this could be when a delivery is received and a sample of the items is taken for quality purposes, if the sample is not sent back to the warehouse within 2 hours, then an alert of missing stock will be generated. These kind of event analysis can also be used to prevent merchandise theft from stores [17], which is also one of the goals of this project. For example, if a product's tag is read in the exit reader, without being read before at the register machine, the system can assume that a possible theft is in progress and alert the security guards.

CEP is an essential tool for the retail supply chain, since it allows the relation of tags to a specific business scenario. It can be used for simple things, like relating the reads of a product tag and a container tag at the same time by the same reader and assume that the product is being shipped inside the container, to the most complicated business rules that a user can introduce, by relating time, cause and place, and trigger an action accordingly [18].

We will be using the ASPIRE project as the base of our work $[19,20]$, since it's design is very close to our needs and especially because it is an Open Source 


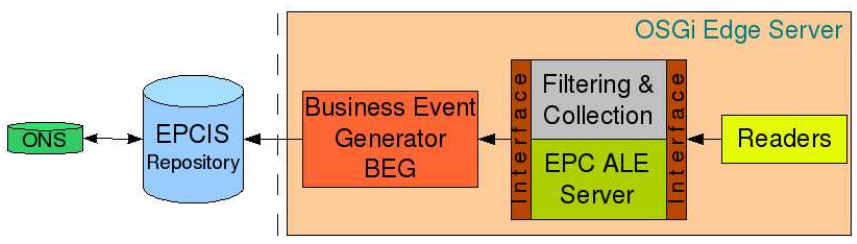

Fig. 5. BEG interaction

solution, which will allow us to adapt it exactly as we need to, and finally it also has an active community, eager to contribute with the adoption and correct use of RFID technologies. The ASPIRE project's specifications include a middleware component that deals with CEP, called Business Event Generator (BEG), a configurable component which captures the raw events sent by the readers and generates new business events [21]. As shown in Figure 5, the BEG receives the filtered events captured by the readers and then queries the EPCIS to get the context information needed for interpreting the incoming data. With all the gathered information, it finds the rules that can be applied to it and then creates the corresponding business event, which in our case will allow the process to move from one state to the other in the process manager.

\subsection{Process manager}

The process manager is the service in charge of relating the business events, created by the BEG, to the business processes. The products follow steps during their life cycle: they are moved, packaged, shipped, received, etc. Those steps are part of a business process. The RFID events are part of the transition between steps in the process. The process manager aims to process the RFID events in order to follow the provided business processes. To implement this part we will use Bonita, a workflow and BPM open source solution [22].

The formal definition of a process allows to specify the expected steps that it will follow and the expected triggers (i.e. RFID events) to go from one step to the other. If an unexpected business event is received at a step where there is no corresponding transition, that means there is an error in the process, either in the design or in the execution of it. All the information obtained from the process (transitions, errors, etc.) can be used by existing software applications, once the information is translated to the format expected by them, which allows an easy adoption of RFID by the companies.

Figure 6 shows an example of a simple process representing the states of a product inside a store. The store is equipped with RFID readers between the areas. A normal process for a product that is In stock, will be to go from the warehouse to the sales floor, after receiving the Leaves stock event. Then it can enter and leave the fitting area, with its corresponding events. Finally, it can go to the cash machine to get sold, which would generate an event to update the stock and then leave the store. 


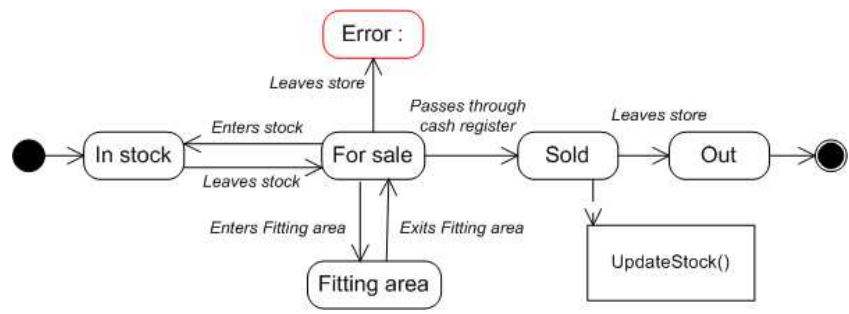

Fig. 6. Store process

When the steps are followed as expected the process continues normally, but when an unexpected event arrives, it means we have a problem. For example, if we receive a Leaves store event, without being in the Sold step, it gets an error and generates an alert. This kind of alerts are an excellent feedback, since they can make the users realize some mistakes in their process design or show them that the process is not being followed as expected, either by the employees or by the clients.

\section{Related work}

A lot of research is being done in different areas, in order to facilitate the adoption of RFID mainly for governments, hospitals and retail suppliers. Most of the recent works propose the use of CEP for obtaining meaningful information from the gathered data and there are different approximations for this topic.

An attempt to apply the principle of separation of concerns in CEP has lead a research to create a layered middleware for it, which consists on a logic structure layer, a temporal constraint layer and an event detection layer [23]. They support their approach by standing that a layered design provides a better understanding for RFID events.

A tool called Cascadia, capable of extracting and managing meaningful highlevel events from raw RFID data is another work on this area [24]. Cascadia offers a complete system for managing RFID events, but it doesn't use any standards for this, so the interaction with other systems depends on specific development for the tool.

A research similar to our project presents a middleware for RFID and Wireless Sensor Networks (WSN), using CEP in a partial EPC architecture, including an EPCIS [25]. They present a good work for monitoring products, considering the environment, but they lack of interaction with an ONS and don't consider traceability in their application.

\section{Conclusions and future work}

Being able to get the present and historic information about a product is very important in the supply chain management, since it helps to make the right deci- 
sions in the right moment. That is why traceability is one of the most important aspects for it, and the lack of research and solutions that successfully implement traceability and persistence has caused the retail supply chain to take longer to adopt RFID. In this paper we presented a service that covers those needs with an easy-to-use tool that will allow the users to exploit the benefits of RFID by integrating it into their business processes, helping them also to get feedback about certain processes that are not being followed as expected.

We propose a service that integrates several existing solutions (like Bonita [22] and ASPIRE [20]) to offer the final users a complete, interesting and easy-touse application. We are still in the process of implementing the service, working with the ASPIRE team and talking with different companies in the retail supply chain to gather their needs and offer solutions.

Acknowledgments - This project is partially funded by the "Pôle de compétitivité" PICOM and the Région Nord-Pas-de-Calais in France.

\section{References}

1. EPCglobal's website. http://www.epcglobalinc.org.

2. Yu-Ju Tu, Wei Zhou, and Selwyn Piramuthu. Identifying RFID-embedded objects in pervasive healthcare applications. Decis. Support Syst., 46(2):586-593, 2009.

3. Chun-Liang Lai, Shou-Wei Chien, Shiu-Ching Chen, and Kwoting Fang. Enhancing medication safety and reduce adverse drug events on inpatient medication administration using RFID. WTOC, 7(10):1045-1054, 2008.

4. Hui Tan. The Application of RFID Technology in the Warehouse Management Information System. In ISECS '08: Proceedings of the 2008 International Symposium on Electronic Commerce and Security, pages 1063-1067, Washington, DC, USA, 2008. IEEE Computer Society.

5. Bo Yan, Yiyun Chen, and Xiaosheng Meng. RFID Technology Applied in Warehouse Management System. In CCCM '08: Proceedings of the 2008 ISECS International Colloquium on Computing, Communication, Control, and Management, pages 363-367, Washington, DC, USA, 2008. IEEE Computer Society.

6. Riaz Ahmad, Fan Yuqing, Zhilun Cheng, and Zou Jihua. Closing information loops with extended PLM. In CSECS'06: Proceedings of the 5th WSEAS International Conference on Circuits, Systems, Electronics, Control $\&$ Signal Processing, pages 344-349, Stevens Point, Wisconsin, USA, 2006. World Scientific and Engineering Academy and Society (WSEAS).

7. Martin Strassner and Elgar Fleisch. The Promise of Auto-ID in the Automotive Industry. Technical report, Auto-ID Center, Massachusetts Institute of Technology.

8. Konstantinos Domdouzis, Bimal Kumar, and Chimay Anumba. Radio-Frequency Identification (RFID) applications: A brief introduction. Adv. Eng. Inform., 21(4):350-355, 2007.

9. Katherine Albrecht. Supermarket Cards: The Tip of the Retail Surveillance Iceberg. Denver University Law Review, 79(4):534-565, 2002.

10. Asghar Sabbaghi and Ganesh Vaidyanathan. Effectiveness and efficiency of RFID technology in supply chain management: strategic values and challenges. J. Theor. Appl. Electron. Commer. Res., 3(2):71-81, 2008. 
11. Yu-Ju Tu and Selwyn Piramuthu. Reducing false reads in RFID-embedded supply chains. J. Theor. Appl. Electron. Commer. Res., 3(2):60-70, 2008.

12. Chin-Ling Chen, Yu-Yi Chen, Yu-Cheng Huang, Chen-Shen Liu, Chia-I Lin, and Tzay-Farn Shih. Anti-counterfeit ownership transfer protocol for low cost RFID system. W. Trans. on Comp., 7(8):1149-1158, 2008.

13. Maria Madlberger. A Model of Antecedents of RFID Adoption Intention in the Supply Chain. In HICSS '09: Proceedings of the 42nd Hawaii International Conference on System Sciences, pages 1-10, Washington, DC, USA, 2009. IEEE Computer Society.

14. E. W. T. Ngai, Karen K. Moon, Frederick J. Riggins, and Candace Y. Yi. RFID research: An academic literature review (1995-2005) and future research directions. International Journal of Production Economics, 112(2):510-520, April 2008.

15. Guangming Wang and Gonglian Jin. Research and Design of RFID Data Processing Model Based on Complex Event Processing. In CSSE '08: Proceedings of the 2008 International Conference on Computer Science and Software Engineering, pages 1396-1399, Washington, DC, USA, 2008. IEEE Computer Society.

16. EPCglobal's Standards. http://www.epcglobalinc.org/standards/.

17. Nicholas Huber and Katina Michael. Minimizing Product Shrinkage across the Supply Chain using Radio Frequency Identification: a Case Study on a Major Australian Retailer. In ICMB '0\%: Proceedings of the International Conference on the Management of Mobile Business, page 45, Washington, DC, USA, 2007. IEEE Computer Society.

18. Tao Ku, YunLong Zhu, and KunYuan Hu. A Novel Complex Event Mining Network for Monitoring RFID-Enable Application. In PACIIA '08: Proceedings of the 2008 IEEE Pacific-Asia Workshop on Computational Intelligence and Industrial Application, pages 925-929, Washington, DC, USA, 2008. IEEE Computer Society.

19. Nikos Kefalakis, Nektarios Leontiadis, John Soldatos, Kiev Gama, and Didier Donsez. Supply chain management and NFC picking demonstrations using the AspireRfid middleware platform. In Companion '08: Proceedings of the ACM/IFIP/USENIX Middleware '08 Conference Companion, pages 66-69, New York, NY, USA, 2008. ACM.

20. ASPIRE's website. http://aspire.objectweb.org.

21. ASPIRE Middleware and Programmability Specifications. http://fp7-aspire. eu/fileadmin/aspire/docs/D2_4.pdf.

22. Bonita's website. http://bonita.objectweb.org.

23. Wenhui Hu, Wei Ye, Yu Huang, and Shikun Zhang. Complex Event Processing in RFID Middleware: A Three Layer Perspective. In ICCIT '08: Proceedings of the 2008 Third International Conference on Convergence and Hybrid Information Technology, pages 1121-1125, Washington, DC, USA, 2008. IEEE Computer Society.

24. Evan Welbourne, Nodira Khoussainova, Julie Letchner, Yang Li, Magdalena Balazinska, Gaetano Borriello, and Dan Suciu. Cascadia: A System for Specifying, Detecting, and Managing RFID Events. In MobiSys '08: Proceeding of the 6th international conference on Mobile systems, applications, and services, pages 281294, New York, NY, USA, 2008. ACM.

25. Weixin Wang, Jongwoo Sung, and Daeyoung Kim. Complex Event Processing in EPC Sensor Network Middleware for Both RFID and WSN. In ISORC '08: Proceedings of the 2008 11th IEEE Symposium on Object Oriented Real-Time Distributed Computing (ISORC), pages 165-169, Washington, DC, USA, 2008. IEEE Computer Society. 